\title{
Controllable Josephson junction for photon Bose-Einstein condensates
}

\author{
Mario Vretenar, Ben Kassenberg, Shivan Bissesar ${ }^{(0)}$, Chris Toebes, and Jan Klaers (1) \\ Complex Photonic Systems (COPS), MESA+ Institute for Nanotechnology, University of Twente, PO Box 217, 7500 AE Enschede, Netherlands
}

(Received 14 January 2021; revised 6 May 2021; accepted 10 May 2021; published 1 June 2021)

\begin{abstract}
Josephson junctions are the basis for the most sensitive magnetic flux detectors, the definition of the unit volt by the Josephson voltage standard, and superconducting digital and quantum computing. They result from the coupling of two coherent quantum states, as they occur in superconductors, superfluids, atomic Bose Einstein Condensates (BECs), and exciton-polariton condensates. In their ground state, Josephson junctions are characterized by an intrinsic phase jump. Controlling this phase jump is fundamental for applications in computing. Here, we experimentally demonstrate controllable phase relations between photon BECs resulting from particle exchange in a thermo-optically tunable potential landscape. Our experiment realizes an optical analog of a controllable $0, \pi$-Josephson junction. By connecting several junctions, we can study a reconfigurable 4-condensate system demonstrating the potential of our approach for analog spin-glass simulation. More generally, the combination of static and dynamic nanostructuring techniques introduced in our work offers a powerful platform for the implementation of adaptive optical systems for paraxial light in and outside of thermal equilibrium.
\end{abstract}

DOI: 10.1103/PhysRevResearch.3.023167

\section{INTRODUCTION}

Finding the energetic ground state of a magnet with disordered couplings is a complicated combinatorial problem. This so-called spin-glass problem has no analytic solution and even numerical techniques are found to be inefficient. It is known that many important optimization problems in machine learning, logistics, computer chip design, and DNA sequencing can be mathematically mapped to an equivalent spin-glass problem [1]. The latter results from the proven NP hardness of the problem [2,3]. A method for solving the spin-glass problem in the Ising, XY, or Heisenberg model can thus serve as a blueprint for approaching a large class of mathematical optimizations. This motivates research on analog spin-glass simulators as a new class of computational devices specifically designed for simulating spin systems. For the Ising model, a first generation of analog spin-glass simulators has been realized using networks of superconducting qubits [4,5] and optical parametric oscillators (OPOs) [6-8]. Whether these simulators already offer a computational advantage over the more conventional von Neumann computer architecture is currently a matter of controversy. For the XY model, the proposed physical platforms are based on superconducting qubits [9], lasers [10], atomic Bose-Einstein condensates (BECs) [11], and polariton condensates [12-17]. The basic idea in these approaches is to associate the XY spins $\varphi_{i} \in[0,2 \pi)$ to the phases of coherent states. A prototypical

Published by the American Physical Society under the terms of the Creative Commons Attribution 4.0 International license. Further distribution of this work must maintain attribution to the author(s) and the published article's title, journal citation, and DOI. spin-glass simulator consists of a lattice of simulated spins, which are coupled to each other in a controllable manner. In the case of coupled BECs, the couplings can be considered as Josephson junctions [18-24]. The ability to accurately adjust the coupling constants between the spins is fundamental for defining the computational problem to be solved. In earlier work with polariton condensates, tunable couplings were realized by controlling the geometrical distance between the condensates or by exploiting polariton-reservoir interactions $[12,13,15,17]$. The former approach is mainly limited to systems with homogeneous couplings across the lattice. Polariton-reservoir and polariton-polariton interactions generally result in a gain [12,15] and time dependence [12] of the condensate couplings. This is undesirable for spinglass simulation as this leads to a continuous redefinition of the computational problem when the system is amplified from the quantum to the classical regime by increasing the optical gain. In this work, we experimentally realize a controllable $0, \pi$-Josephson junction for photon BECs exploiting the anomalously large thermo-optical coefficient of an optical medium close to a phase transition. By connecting several junctions, we can study effective 4-spin systems demonstrating the potential of our approach for analog spin-glass simulation.

\section{EXPERIMENTAL SYSTEM}

Our experimental setup is based on a high-finesse dye microcavity, see Fig. 1(a), in which optical photons propagate paraxial to the optical axis ( $z$ axis) and are repeatedly absorbed and reemitted by dye molecules. The dye molecules obey the Kennard-Stepanov (KS) law connecting the (broadband) Einstein coefficients of absorption $B_{12}(\omega)$ and emission $B_{21}(\omega)$ by a Boltzmann factor: $B_{12}(\omega) / B_{21}(\omega)=\exp [\hbar(\omega-$ 
(a)

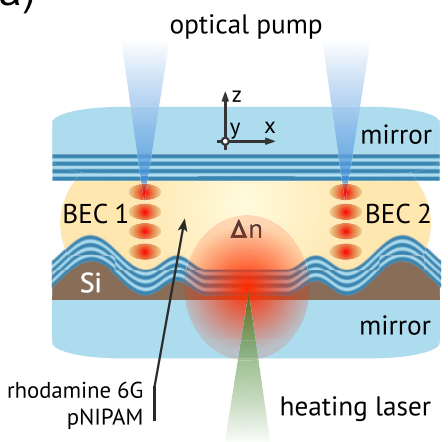

(b)

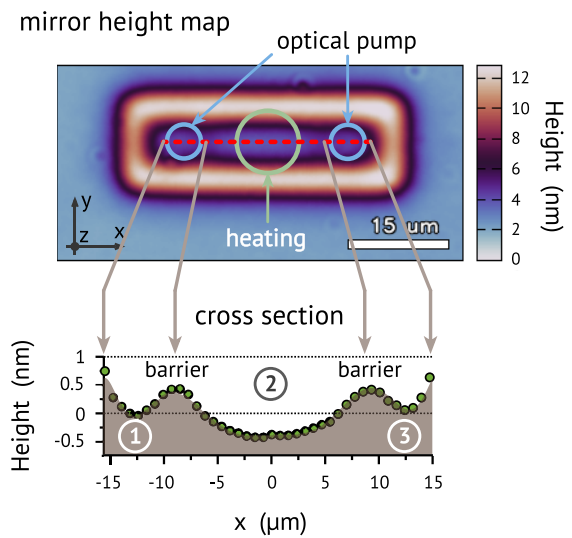

(c)

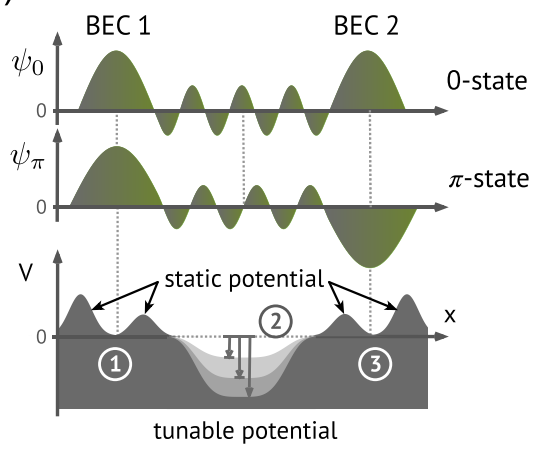

FIG. 1. Controllable Josephson junction for photon BECs. (a) The experimental setup is based on a high-finesse dye microcavity, in which optical photons propagate paraxial to the optical axis ( $z$ axis) and are repeatedly absorbed and reemitted by dye molecules. The latter leads to a thermalization and condensation of the photon gas at room temperature. The potential landscape for the two-dimensional photon gas is created by a combination of two experimental techniques: A static nanostructuring of one of the mirror surfaces and an in situ variation of the index of refraction of the optical medium using a thermo-responsive polymer (pNIPAM) and a heating laser. (b) Static confinement potential. A rectangular surface structure is created on one of the cavity mirrors, which restricts the flow of light to the confined area. This area is furthermore divided into three parts by introducing two shallower barriers; see the cross-section in the lower graph. The height map of the mirror was determined by Mirau interferometry. (c) The Josephson junction in our experiment consists of two photon BECs that are created in local potential minima at the two ends of the junction. By tunneling into the central region of the junction, a particle exchange is created that leads to in-phase or anti-phase relations between the condensates (upper two graphs). The acquired phase delay is controlled by the thermo-optically induced potential (lower graph).

$\left.\left.\omega_{\text {zpl }}\right) / k T\right]$. Here, $\omega_{\text {zpl }}$ is the zero-phonon line of the dye and $T$ is the temperature. Multiple absorption-emission cycles establish a thermal contact between the photon gas and its environment, which, in effect, leads to a thermalization and condensation of the photon gas at room temperature [25-32]. To achieve that, the rate of photon absorption by the optical medium needs to be significantly higher than the rate of photon transmission through the mirrors. The latter distinguishes photon condensation experiments from conventional lasing phenomena. For sufficiently small mirror spacing, the photon gas effectively becomes two-dimensional and follows a modified energy-momentum relation given by

$$
E \simeq \frac{m c^{2}}{n_{0}^{2}}+\frac{\left(\hbar k_{r}\right)^{2}}{2 m}-\frac{m c^{2}}{n_{0}^{2}}\left(\frac{\Delta d}{D_{0}}+\frac{\Delta n}{n_{0}}\right),
$$

where $k_{r}$ represents the transverse wave number and $m$ denotes an effective photon mass (see Appendix A). The second and third terms correspond to the kinetic and potential energy of the photons. The potential energy is nonvanishing, if the distance between the mirrors $D(x, y)=D_{0}+\Delta d(x, y)$ or the refractive index $n(x, y)=n_{0}+\Delta n(x, y)$ is modified across the transverse plane of the resonator. In our derivation, we assume $\Delta d \ll D_{0}$ and $\Delta n \ll n_{0}$.

Controlling the potential landscape within the microresonator through static and dynamic nanostructuring techniques is fundamental to this experiment. Various experimental techniques for shaping the potential energy profiles in microcavity systems have been developed, for example, methods based on strain [33], surface waves [34], electrostatic [35] and exciton reservoir confinement [36], and deep etching [37]. The Josephson junction in our experiment consists of two photon BECs that exchange particles in a tunable potential landscape created by a combination of two experimental techniques: Nanostructuring of the mirror surface and in situ variation of the index of refraction of the optical medium. For the static nanostructuring, we use a novel direct laser writing technique that allows us to selectively lift the surface of the mirror by several hundred nanometers with subnanometer control [38]. This translates into repulsive potentials for the photon gas in a microresonator setting; see Eq. (1).

In a first step, we create a barrier around a rectangular region of $30 \mu \mathrm{m}$ length and $5 \mu \mathrm{m}$ width, see Fig. 1(b), which restricts the flow of light to the confined area. In a second step, we divide the confined area into three regions by introducing two shallower barriers [the three regions are denoted by 1 , 2, and 3 in Figs. 1(b) and 1(c)]. The microresonator setup is completed by a second (planar) mirror and an optical medium, which is a water-based solution of rhodamine $6 \mathrm{G}$ and the thermo-responsive polymer pNIPAM. By optically pumping the dye molecules with a 5-ns laser pulse at $\lambda=480 \mathrm{~nm}$, we create photon BECs in local potential minima at the two ends of the structure. The finite potential barriers allow photons to tunnel into the central region of the junction establishing a particle exchange between the two condensates. The thermo-responsive polymer pNIPAM is used to control the potential landscape in the central region of the junction. For that, we use a second laser that emits 2-ms-long laser pulses of variable energy. These pulses are irradiated onto the sample $50 \mathrm{~ms}$ before the optical pumping initiates the condensation process. The pulses are absorbed in an amorphous silicon layer located below the dielectric stack of one of our mirrors [Si in Fig. 1(a)]. The absorbed energy increases the local temperature of the optical medium by a few Kelvin such that it reaches the lower critical solution temperature (LCST) of 
(a)

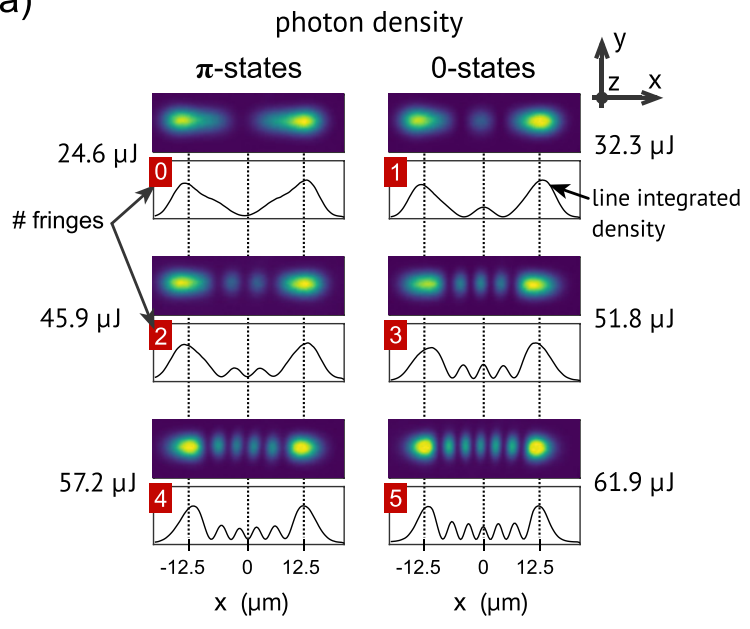

(b)

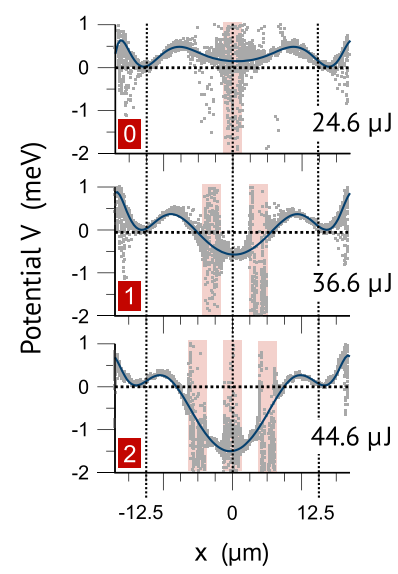

(c)

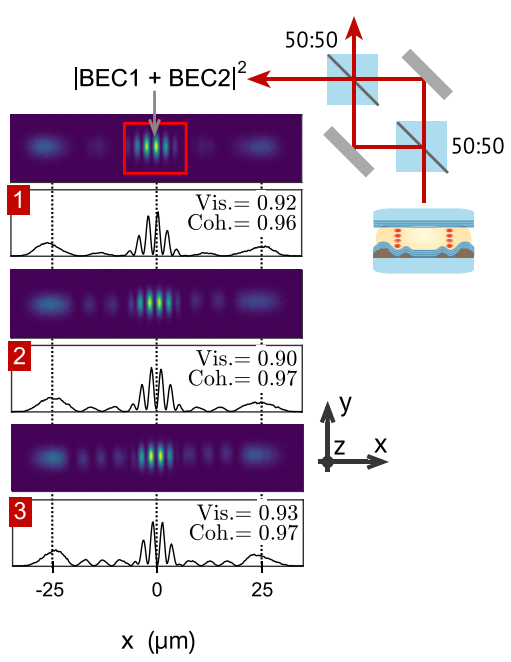

FIG. 2. Controllable phase relations. (a) Photon density in the microresonator plane for six different heating energies as determined by a camera capturing the light transmitted by one of the cavity mirrors. All density profiles can be assigned to symmetric wave functions ( 0 -states) or antisymmetric wave functions ( $\pi$-states). The numbers in the red boxes indicate the number of observed intensity maxima between the condensates. (b) Potential landscape in the microresonator. Starting from the measured photon density $\rho=|\psi(x, y)|^{2}$, we reconstruct the wave function of the photons $\psi(x)=\epsilon(x) \sqrt{\rho(x)}$, in which $\epsilon(x)$ switches between +1 and -1 at every node in the wave function. The potential $V$ follows via $V-$ const $=-E_{\text {kin }}=\left(\hbar^{2} / 2 m\right)\left(d^{2} \psi / d x^{2}\right) \psi^{-1}$ (points). Our method breaks down at the nodes of the wave function. These regions (shaded areas) are excluded from the polynomial fit (line). The reconstructed potentials reveal both the static potential due to the nanostructured mirror and the dynamically induced potential due to the thermo-responsive polymer. (c) Interferometric images of the coupled condensate system. The transmitted light is guided through a Mach-Zehnder interferometer to test for the coherence of the two condensates. Data for the interferometric visibility $v=\left(I_{\max }-I_{\min }\right) /\left(I_{\max }+I_{\min }\right)$ are given in the inset. The estimated degree of first-order coherence, which is generally found to be close to unity, takes into account the (small) population imbalances between the condensates.

pNIPAM in water $\left(32^{\circ} \mathrm{C}\right)$. This leads to a significant change of the index of refraction which, with Eq. (1), translates into a tunable potential for the photons in the microcavity [28]. The purpose of this tunable potential is to control the phase delay that the photons acquire while traveling through the central region of the junction; see Fig. 1(c).

The coupled photon BECs system follows equations of motion that closely resemble the Josephson equations (see Appendix B). Deviations from the textbook Josephson scenario arise from the interaction with the environment. In the photon BECs system, the states of the condensates are not given entities but are subject to energy and particle exchange with the environment. In particular, the evolution of the total photon number $n=n_{1}+n_{2}$ is equal to

$$
\dot{n}=\lambda n-J_{12} \sqrt{n_{1} n_{2}} \cos \left(\theta_{1}-\theta_{2}\right),
$$

where $n_{1,2}$ and $\theta_{1,2}$ are the photon numbers and phases of the two condensates, $\lambda$ denotes a gain parameter, and $J_{12}$ is the coupling between the two condensates (see Appendix B). Optically pumping the system triggers the condensation process and initiates a competition among the possible system states. In this competition, the state that maximizes the gain $\dot{n}$ is the one that acquires the dominant statistical weight. Based on Eq. (2), we expect that the system predominantly realizes states with equal condensate population (as this maximizes the square root function for a given $n$ ) and phase differences of $\theta_{1}-\theta_{2}=0$ ( 0 -state) or $\theta_{1}-\theta_{2}=\pi$ ( $\pi$-state) depending on the sign of $J_{12}$. The latter is a function of the induced potential and other system parameters (see Appendix B). These two states maximize the gain, are fixed points of the Josephson equations, and thus define the intrinsic phase jump across the junction.

\section{RESULTS}

\section{A. Single junction}

Figure 2(a) shows the photon density $\rho=|\psi(x, y)|^{2}$ inside the microresonator as determined by a camera capturing the light transmitted by one of the cavity mirrors. We observe various stripe patterns indicating the presence of photon exchange and the formation of standing waves between the condensates. All density profiles can be clearly assigned to symmetric wave functions (0-states) or antisymmetric wave functions ( $\pi$-states); see Fig. 1(c). With increasing potential depth, symmetric and antisymmetric states alternate and the number of nodes in the wave function increases. The measured photon density $\rho$ allows us to reconstruct the potential landscape in the microresonator. Using $V-$ const $=-E_{\mathrm{kin}}=$ $\left(\hbar^{2} / 2 m\right)\left(d^{2} \psi / d x^{2}\right) \psi^{-1}$ with $\psi(x)=\epsilon(x) \sqrt{\rho(x)}$ and a sign function $\epsilon(x)$ switching between +1 and -1 at every node, we obtain the spatial variation of the potential in the $x$-direction of the microcavity plane; see Fig. 2(b). The reconstructed potentials clearly reveal both the static potential due to the nanostructured mirror and the dynamically induced potential due to the thermo-responsive polymer. This confirms that the condensation takes place in the ground states of local energy minima. Condensation in the global potential energy minimum (located between the two condensates) is intentionally prevented by the applied inhomogeneous optical pumping. Interferometric images of the coupled condensate system are shown in Fig. 2(c). For this, we guide the 
(a)

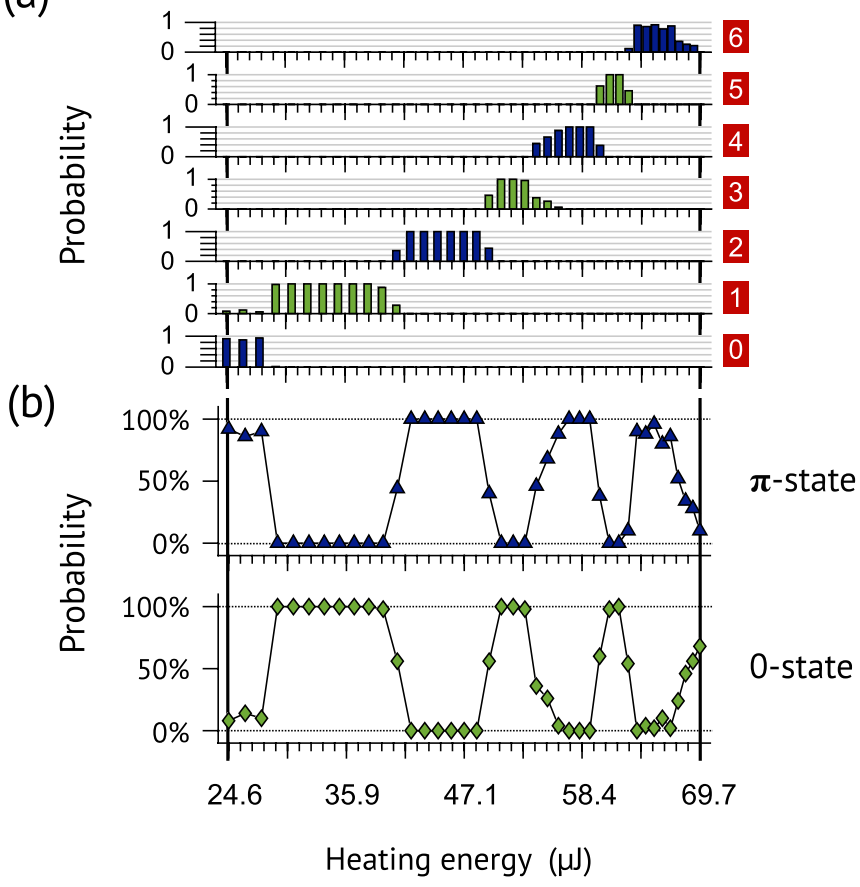

FIG. 3. State-resolved statistics. (a) State-resolved statistical information on the operation of the junction. For every heating energy, 50 experimental runs with identical control parameters are measured and analyzed. The data indicate that the formation of a particular junction state, as defined by the number of observed intensity maxima between the condensates (red boxes), is deterministic for a significant fraction of the investigated heating energies. (b) Probability of finding the junction in a 0 - or $\pi$-state as a function of the heating energy. transmitted light through a Mach-Zehnder interferometer, see the schematic representation in Fig. 2(c), and superimpose the images of the two condensates. The high contrast of the observed stripe pattern indicates first-order coherences close to unity. The fact that this level of coherence is reached when integrating over one Gaussian pump pulse proves that the coupling in our junction is neither dependent on optical gain nor influenced by self-interactions. The latter is an essential requirement for using the junction in optical spin-glass simulation. State-resolved statistical information on the operation of the junction is provided in Fig. 3(a). Here we measure and analyze sequences of 50 experimental runs with identical control parameters. The data indicate that the formation of a particular phase relation between the condensates is deterministic for a significant fraction of the investigated heating energies. Moreover, the transition between the states is quite sharp; see Fig. 3(b).

\section{B. Multiple junctions}

When several junctions are connected together, the gain function Eq. (2) can be generalized to $\dot{n}=\sum_{i} \lambda n_{i}-H_{X Y}$ with $H_{X Y}=-\sum_{i>j} J_{i j}^{X Y} \cos \left(\theta_{i}-\theta_{j}\right)$ and $J_{i j}^{X Y}=-J_{i j} \sqrt{n_{i} n_{j}}$. Maximizing the gain thus corresponds to minimizing the energy of a simulated XY system with couplings $J_{i j}^{X Y}$, which demonstrates the connection with the spin-glass problem (see also Appendix B). Figure 4(a) shows a mirror surface profile that is designed to implement four mutually coupled Josephson junctions with rectangular symmetry. When the four corners of the structure are pumped optically, condensates form and establish phase relations with one another. By triggering the polymer phase transition, we can switch between positive (in-phase) and negative (anti-phase) (a)

mirror height map
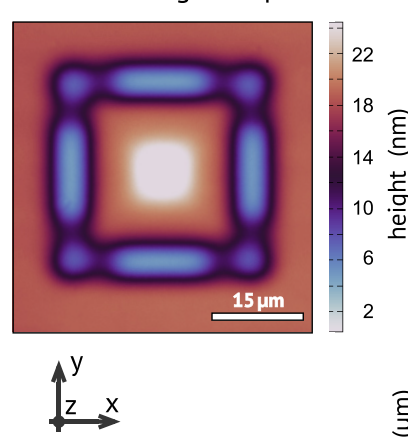

(b)

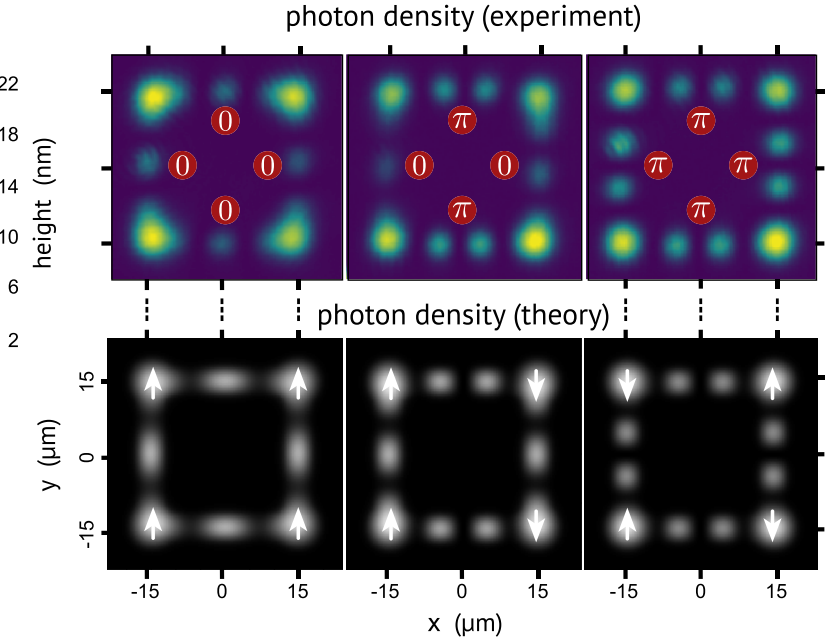

(c)

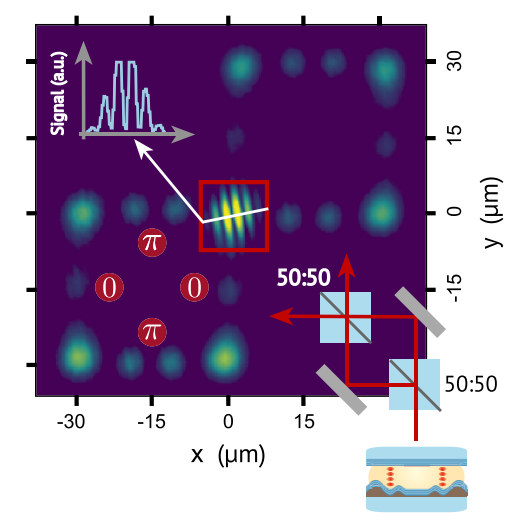

FIG. 4. Higher-order optical circuit composed of four Josephson junctions. (a) Height map of the used cavity mirror. Optical pumping with nanosecond pulses is carried out at the four corners of the structure. (b) Photon density for three different coupling configurations as denoted by the labels 0 and $\pi$. By varying the spatial heat profile in the microcavity, the phase relations of the four condensates can be switched between inphase and anti-phase. The top panel shows experimental results, while the bottom panel contains theoretical results derived from the numerical solution of a driven-dissipative Schrödinger equation (see Appendix B). The white arrows correspond to the phases of the photonic wave function at the position of the condensates and can be interpreted as the angular orientation of four XY spins. (c) Interferometric imaging of the four coupled condensates using a Mach-Zehnder interferometer. The top right condensate in one interferometer path is superimposed on the bottom left condensate in the other interferometer path. The observed interference fringes indicate coherence close to unity (see cross-section). 
(a)

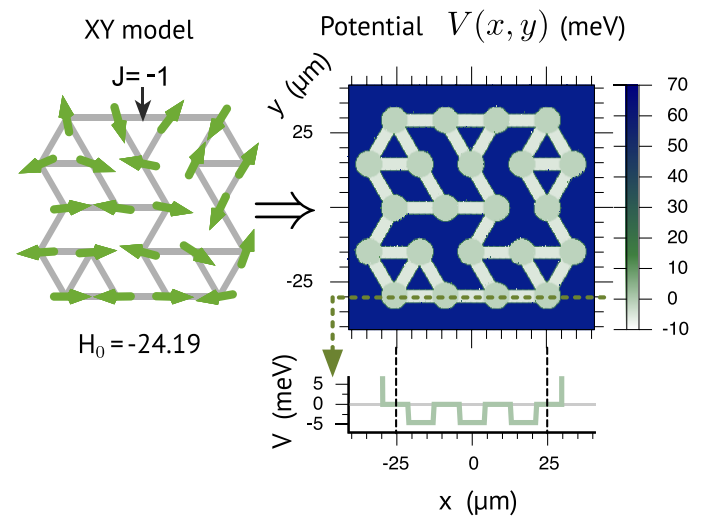

(b) Amplitude $|\psi(x, y)|$ (a.u.)

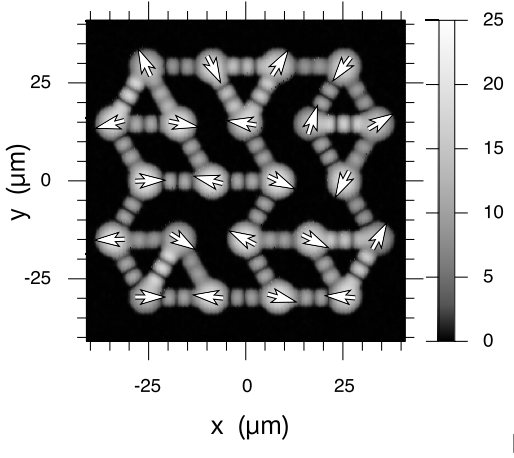

(c)

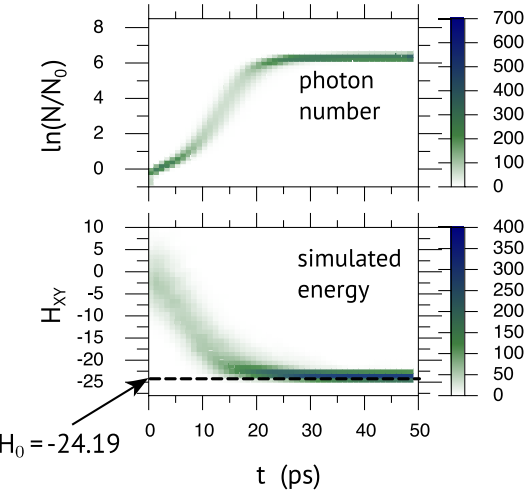

FIG. 5. Proposed scheme for optical spin-glass simulation with photonic Josephson junctions. (a) Mapping between XY spin glasses and systems of coupled BECs. We propose to use photonic Josephson junctions to solve optimization problems, namely the ground-state problem in XY spin glasses and mathematical equivalent problems. The ground-state problem corresponds to finding the minimum of $H_{X Y}=$ $-\sum_{i>j} J_{i j} \cos \left(\phi_{i}-\phi_{j}\right)$ with optimization variables $\phi_{i} \in[0,2 \pi)$. A particular instance of such a problem is defined on a (random) 3-regular planar graph with antiferromagnetic couplings $J_{i j}=-1$. The problem is mapped onto the potential landscape in the microcavity system by introducing a triangular lattice potential, in which the lattice sites are connected by attractive step potentials. The depth of the steps is chosen to favor the formation of $\pi$-states. (b) Amplitude of the simulated light field $|\psi(x, y)|$. We have performed numerical simulations to investigate the condensation process in the given potential landscape. A two-dimensional stochastic Schrödinger equation with gain and frequency-dependent loss terms is numerically integrated using the Runge-Kutta method (see Appendix B). At the start of the simulation, the optical gain on the triangular lattice is set close to the condensation threshold and photons start to populate the cavity. The phases of the light field, indicated by the arrows, are adjusted to maximize the gain. (c) Total photon number and simulated energy as a function of time. The data comprise 300 numerically obtained stochastic time evolutions of the system. The relative frequency of the total photon number $N$ and the simulated energy $H_{X Y}$, derived from the phases of the light field, are color coded. The photon number is normalized to the noise level $N_{0}$ in the stochastic Schrödinger equation (at zero gain). The coupled condensate system is capable of finding good approximative solutions of the given ground-state problem within a period as short as $20 \mathrm{ps}$.

couplings in this structure. Three different states of the condensate lattice, which result from three different heat patterns applied to the thermo-responsive optical medium, are shown in Fig 4(b). In all cases, we have verified that the four condensates are coherent to each other, as exemplarily shown in Fig. 4(c). The observed states can be interpreted as the solutions to three different ground-state problems defined in a 4-spin XY model.

\section{DISCUSSION}

We expect that analog spin-glass simulation will be the first major application of photonic Josephson junctions. To illustrate the potential of this approach, we propose and numerically analyze a scheme for solving the ground-state problem in XY spin glasses using networks of coupled photon BECs. A particular instance of such a problem is defined in Fig. 5(a), which shows a classical XY model with Hamiltonian $H_{X Y}=-\sum_{i>j} J_{i j} \cos \left(\phi_{i}-\phi_{j}\right)$ on a (random) 3-regular planar graph with antiferromagnetic couplings $J_{i j}=-1$. As discussed before, the basic idea is to associate the XY spins $\phi_{i} \in[0,2 \pi)$ to the phases $\theta_{i}$ of photon BECs. For the given example, we assume a triangular lattice potential in the microcavity. The lattice sites are connected to each other by attractive step potentials with variable depth. For mapping the $\mathrm{XY}$ coupling constants $J_{i j}=-1$ to the photon BECs system, we set the potential depth to a value that favors the formation of $\pi$-states. This results in the potential landscape shown in Fig. 5(a). We have performed numerical simulations to investigate the dynamics of the condensation process in the given potential landscape. For this, we numerically integrate a two-dimensional stochastic Schrödinger equation with gain and frequency-dependent loss terms in an experimentally relevant parameter regime (see Appendix B). At $t=0$, the optical gain on the triangular lattice is set close to the condensation threshold and photons start to populate the cavity. This starts a competition among the various system states, in which the phases of the light field, indicated by the arrows in Fig. 5(b), are adjusted to maximize the gain function. As discussed before, the latter is a straightforward generalization of Eq. (2) and can be shown to coincide with the XY Hamiltonian $H_{X Y}$ (see Appendix B). Figure 5(c) shows the total photon number and the simulated energy, derived from the phases of the light field, as a function of time. The data comprise 300 numerically obtained stochastic time evolutions of the system. The relative frequency of photon number and simulated energy are color coded. From this figure, we conclude that the coupled condensate system is capable of finding good approximative solutions of the given ground-state problem within $30 \mathrm{ps}$. This is faster than a single tick of the clock in a conventional CPU, which gives an indication of the potential of optical spinglass simulation. That said, we would like to emphasize that many aspects determining the performance of the proposed simulator will need to be investigated more closely in the 
future and several technological challenges have yet to be overcome before such investigations can begin.

\section{CONCLUSION}

In conclusion, our work introduces and investigates Josephson junctions for photon BECs controlled by thermooptical interactions. We expect that photonic Josephson junctions can play a major role for novel computational schemes, for example, in analog spin-glass simulation and oscillatory neural networks. Various approaches to realizing such simulators are currently being investigated. At this time it is not clear whether the first generation of spin-glass simulators provides a computational advantage over the more conventional von Neumann computer architecture. In the different approaches there are also different challenges that have to be overcome, for example, the replacement of digital electronic components in the computation process, miniaturization, nonlocal couplings, and scaling. At the moment, we do not see a system that has overcome all of these challenges. For the photon BECs system, scaling to larger system sizes is the most challenging aspect. In general, phase-coherent coupling of hundreds or even thousands of coherent states is technically feasible and has already been achieved for polaritonic BECs [15,39] and laserlike systems [10]. However, controllable couplings with single bond resolution have not yet been realized in such systems and remain a challenge. The research on Bose-Einstein condensation of photons has brought forth experimental techniques that allow an unprecedented level of control of microresonators [28,38]. Based on the results presented in this work, we believe that these techniques are suitable for overcoming this challenge. Beyond the application in spin-glass simulation, the combination of static nanostructuring with a thermo-responsive optical medium introduced in this work moreover offers a powerful platform for the implementation of adaptive optical circuits, lattice systems, and photonic crystals for paraxial light.

\section{ACKNOWLEDGMENTS}

We thank Klaas-Jan Gorter for his contributions in the early phase of this project and the Weitz group at the University of Bonn for providing critical equipment. Useful discussions with Natalia Berloff, Pavlos Lagoudakis, Michiel Wouters, Pepijn Pinkse, and Willem Vos are acknowledged. This work has received funding from the European Research Council (ERC) under the European Union's Horizon 2020 research and innovation programme (Grant Agreement No. 101001512).

\section{APPENDIX A: EXPERIMENTAL METHODS}

\section{Microcavity setup}

Our experiment is based on a high-finesse microcavity as shown in Fig. 1(a) of our paper. In this system, photons behave as two-dimensional massive particles subject to a controllable potential energy landscape. In general, the photon energy in the cavity is given by $E=(\hbar c / n) \sqrt{k_{z}^{2}+k_{r}^{2}}$, in which the longitudinal (along the optical axis) and transverse wave numbers are denoted by $k_{z}$ and $k_{r}$. The boundary conditions induced by the mirrors require $k_{z}=q \pi / D(x, y)$, in which $q=\mathrm{const}$ is the longitudinal mode number and $D(x, y)$ denotes the mirror separation. We allow small variations in the index of refraction $n(x, y)=n_{0}+\Delta n(x, y)$ and in the mirror separation $D(x, y)=D_{0}+\Delta d(x, y)$ across the transverse plane of the resonator. Assuming $k_{r} \ll k_{z}, \Delta n \ll n_{0}$ and $\Delta d \ll D_{0}$, we can approximate the photon energy by

$$
E \simeq \frac{m c^{2}}{n_{0}^{2}}+\frac{\left(\hbar k_{r}\right)^{2}}{2 m}-\frac{m c^{2}}{n_{0}^{2}}\left(\frac{\Delta d}{D_{0}}+\frac{\Delta n}{n_{0}}\right),
$$

in which only leading-order contributions were retained. Furthermore, we have defined an effective photon mass $m=$ $\pi \hbar n_{0} q / c D_{0}$. The first term corresponds to the rest energy of the (two-dimensional) photon. The second term is the kinetic energy and the third term corresponds to the potential energy of the photon. The latter is nonvanishing, if the index of refraction or the mirror distance varies across the transverse plane of the resonator. In our experiment, the index of refraction is controlled by means of a thermo-responsive polymer [28] and variations of the mirror distance are achieved by nanostructuring one of the mirrors with a direct laser writing technique [38]. The height profiles of our mirrors are determined via Mirau interferometry. For the latter, we use a commercially available interferometric microscope objective $(20 \times$ Nikon CF IC Epi Plan DI).

In our experiment, the rest energy of the photon is $m c^{2} / n_{0}^{2} \simeq 2.1 \mathrm{eV}$ (yellow spectral regime). The mirror separation $D_{0}$ can be determined using Eq. (A1), which relates height differences to differences in potential energy: $\Delta V=-\left(m c^{2} / n_{0}^{2}\right) \Delta d / D_{0}$. Based on Figs. 1(b) and 2(b), we conclude that a height difference of $\Delta d \simeq 0.45 \mathrm{~nm}$ is accompanied by a potential difference of $\Delta V \simeq 0.4 \mathrm{meV}$ (barrier of the condensate confinement). The latter corresponds to a mirror separation of $D_{0}=-\left(m c^{2} / n_{0}^{2}\right) \Delta d / \Delta V \simeq 2.3 \mu \mathrm{m}$, which is similar to earlier experiments in the photon BECs system $[25,26]$.

\section{Optical medium}

The optical medium in our experiment is a water-based solution of rhodamine $6 \mathrm{G}$ (concentration $1 \mathrm{mmol} / \mathrm{l}$ ) and the thermo-responsive polymer pNIPAM (4\% mass fraction). To avoid self-quenching of the dye molecules, we add a small amount of lauryldimethylamine $\mathrm{N}$-oxide. Due to the addition of pNIPAM, the index of refraction of our optical medium is expected to be slightly higher than that of pure water.

A necessary condition for the occurrence of photon BoseEinstein condensation in an optical medium is the KS law. The KS law describes the temperature dependence of absorption $B_{12}(\omega)$ and emission $B_{21}(\omega)$ coefficient of a broadband fluorescent medium such as a dye solution:

$$
\frac{B_{12}(\omega)}{B_{21}(\omega)}=e^{\frac{\hbar\left(\omega-\omega_{2 \mathrm{pl}}\right)}{k T}} .
$$

Here, $\omega_{\mathrm{zpl}}$ denotes the zero-phonon line and $T$ describes the temperature of the medium (all experiments were carried out at room temperature). The KS law goes back to a subpicosecond thermalization process of the vibrational and rotational states of the dye by collisions with solvent molecules and is well fulfilled for the dye species in our experiment. It can be 
shown that multiple absorption-emission cycles drive a photon gas toward thermal equilibrium with the optical medium [40]. The KS law is fulfilled if the emission coefficient is constant, i.e., $B_{21}(\omega)=\hat{B}$, and the absorption coefficient increases exponentially with the photon frequency, i.e., $B_{12}=$ $\hat{B} \exp \left(\hbar\left(\omega-\omega_{\text {zpl }}\right) / k T\right)$. This condition is used in our theoretical modeling and approximately corresponds to the situation that is realized in experiments.

For optically exciting the dye molecules in the microcavity, we use a pulsed OPO emitting at a wavelength of $480 \mathrm{~nm}$ with a pulse duration of $5 \mathrm{~ns}$. The heating of the thermo-responsive polymer is achieved with a 2-ms laser pulse at a wave length of $532 \mathrm{~nm}$. The heating pulse is irradiated onto the sample $50 \mathrm{~ms}$ before the optical pump pulse. The heating energy is absorbed in an amorphous silicon layer located below the dielectric stack of one of our mirrors. This leads to an increase of the local temperature of the optical medium by a few Kelvin such that it reaches the LCST of pNIPAM in water. The water molecules attached to the polymer chains are set free and the polymer chains collapse. This initiates a mass transport that leads to a significant change of the index of refraction, which, with Eq. (1), translates into a tunable potential for the photons in the microcavity [28].

\section{APPENDIX B: THEORETICAL METHODS}

\section{Josephson equations for coupled photon BECs}

The system of two coupled condensates with gain and frequency-dependent loss is sketched in Fig. 6. It can be described by two stochastic and dissipative Schrödinger equations,

$$
\begin{aligned}
i \hbar \dot{\psi}_{1}= & \hbar \omega_{c, 1} \psi_{1}+\frac{i \hbar}{2}\left\{\Gamma_{\uparrow, 1}-\Gamma_{\downarrow} \exp \left[\frac{\hbar\left(\dot{\theta}_{1}-\omega_{\text {zpl }}\right)}{k T}\right]\right\} \psi_{1} \\
& +\hbar J \psi_{2}+\hbar \eta_{1} \psi_{1} \\
i \hbar \dot{\psi}_{2}= & \hbar \omega_{c, 2} \psi_{2}+\frac{i \hbar}{2}\left\{\Gamma_{\uparrow, 2}-\Gamma_{\downarrow} \exp \left[\frac{\hbar\left(\dot{\theta}_{2}-\omega_{\text {zpl }}\right)}{k T}\right]\right\} \psi_{2} \\
& +\hbar J \psi_{1}+\hbar \eta_{2} \psi_{2} .
\end{aligned}
$$

Here, $\omega_{c, 1}$ and $\omega_{c, 2}$ describe the bare condensate frequencies. The numerical constants $\Gamma_{\uparrow, 1}, \Gamma_{\uparrow, 2}$, and $\Gamma_{\downarrow}$ describe gain and loss rates, while $J$ denotes the coupling of the two

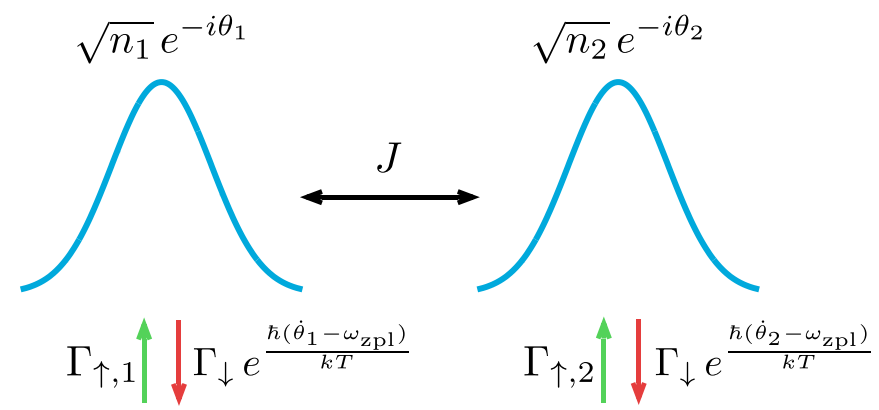

FIG. 6. Two coupled photon BECs with frequency-dependent gain-loss scheme. The latter follows the KS law for fluorescent media and is responsible for a thermalization process between the coupled condensate system and its environment. condensates. In general, $J$ can be a complex number. The equations include noise terms $\hbar \eta_{1,2} \psi_{1,2}$ with noise functions $\eta_{1,2}=\eta_{1,2}(t)$. This renders the time evolution of the system stochastic. The functions $\theta_{1,2}$ denote the phases in the wave functions

$$
\psi_{1,2}=\sqrt{n_{1,2}} \exp \left(-i \theta_{1,2}\right) .
$$

We now define the center frequency of the condensates, namely $\bar{\omega}_{c}=\left(\omega_{c, 1}+\omega_{c, 2}\right) / 2$, and rewrite the absorption term in Eqs. (B1) and (B2) as

$$
\Gamma_{\downarrow} \exp \left[\frac{\hbar\left(\dot{\theta}_{1,2}-\omega_{\mathrm{zpl}}\right)}{k T}\right]=\tilde{\Gamma}_{\downarrow} \exp \left[\frac{\hbar\left(\dot{\theta}_{1,2}-\bar{\omega}_{c}\right)}{k T}\right],
$$

with $\tilde{\Gamma}_{\downarrow}=\Gamma_{\downarrow} \exp \left[\hbar\left(\bar{\omega}_{c}-\omega_{\text {zpl }}\right) / k T\right]$. Assuming that all occurring frequencies remain close to $\bar{\omega}_{c}$, i.e., $\hbar\left|\dot{\theta}_{1,2}-\bar{\omega}_{c}\right| \ll$ $k T$, we can linearize the exponential function in the gain-loss term. This leads to

$$
\begin{aligned}
i \hbar \dot{\psi}_{1}= & \hbar \omega_{c, 1} \psi_{1}+\frac{i \hbar}{2}\left[\tilde{\Gamma}_{\uparrow, 1}-\tilde{\Gamma}_{\downarrow} \frac{\hbar\left(\dot{\theta}_{1}-\bar{\omega}_{c}\right)}{k T}\right] \psi_{1} \\
& +\hbar J \psi_{2}+\hbar \eta_{1} \psi_{1} \\
i \hbar \dot{\psi}_{2}= & \hbar \omega_{c, 2} \psi_{2}+\frac{i \hbar}{2}\left[\tilde{\Gamma}_{\uparrow, 2}-\tilde{\Gamma}_{\downarrow} \frac{\hbar\left(\dot{\theta}_{2}-\bar{\omega}_{c}\right)}{k T}\right] \psi_{2} \\
& +\hbar J \psi_{1}+\hbar \eta_{2} \psi_{2} .
\end{aligned}
$$

In the latter, we have introduced the renormalized gain coefficients $\tilde{\Gamma}_{\uparrow, 1}=\Gamma_{\uparrow, 1}-\tilde{\Gamma}_{\downarrow}$ and $\tilde{\Gamma}_{\uparrow, 2}=\Gamma_{\uparrow, 2}-\tilde{\Gamma}_{\downarrow}$. Using the temporal derivative of Eq. (B3), we replace the phase velocities via

$$
\dot{\theta}_{1,2}=i\left(\frac{\dot{\psi}_{1,2}}{\psi_{1,2}}-\frac{\dot{n}_{1,2}}{2 n_{1,2}}\right) .
$$

Again using Eq. (B3) and separating into real and imaginary parts (Madelung transformation), we arrive at the Josephson equations for the coupled photon BECs system. For the evolution of the condensate phases we find

$$
\begin{aligned}
\dot{\theta}_{1}= & \omega_{c, 1} \operatorname{Re}(\mathcal{A})-\bar{\omega}_{c}[\operatorname{Re}(\mathcal{A})-1]+\frac{1}{2} \operatorname{Im}(\mathcal{A})\left(\frac{\dot{n}_{1}}{n_{1}}-\tilde{\Gamma}_{\uparrow, 1}\right) \\
& +|\mathcal{A}||J| \sqrt{\frac{n_{2}}{n_{1}}} \cos \left(\theta_{J}+\theta_{\mathcal{A}}+\delta\right)+\operatorname{Re}\left(\mathcal{A} \eta_{1}\right) \\
\dot{\theta}_{2}= & \omega_{c, 2} \operatorname{Re}(\mathcal{A})-\bar{\omega}_{c}[\operatorname{Re}(\mathcal{A})-1]+\frac{1}{2} \operatorname{Im}(\mathcal{A})\left(\frac{\dot{n}_{2}}{n_{2}}-\tilde{\Gamma}_{\uparrow, 2}\right) \\
& +|\mathcal{A}||J| \sqrt{\frac{n_{1}}{n_{2}}} \cos \left(\theta_{J}+\theta_{\mathcal{A}}-\delta\right)+\operatorname{Re}\left(\mathcal{A} \eta_{2}\right) .
\end{aligned}
$$

For the evolution of the particle numbers we derive

$$
\begin{aligned}
\dot{n}_{1}= & {\left[\tilde{\Gamma}_{\uparrow, 1}+2 \frac{\operatorname{Im}(\mathcal{A})}{\operatorname{Re}(\mathcal{A})}\left(\omega_{c, 1}-\bar{\omega}_{c}\right)\right] n_{1} } \\
& +\frac{2|\mathcal{A}||J|}{\operatorname{Re}(\mathcal{A})} \sqrt{n_{1} n_{2}} \sin \left(\theta_{J}+\theta_{\mathcal{A}}+\delta\right)+2 \operatorname{Im}\left(\mathcal{A} \eta_{1}\right) n_{1} \\
\dot{n}_{2}= & {\left[\tilde{\Gamma}_{\uparrow, 2}+2 \frac{\operatorname{Im}(\mathcal{A})}{\operatorname{Re}(\mathcal{A})}\left(\omega_{c, 2}-\bar{\omega}_{c}\right)\right] n_{2} } \\
& +\frac{2|\mathcal{A}||J|}{\operatorname{Re}(\mathcal{A})} \sqrt{n_{1} n_{2}} \sin \left(\theta_{J}+\theta_{\mathcal{A}}-\delta\right)+2 \operatorname{Im}\left(\mathcal{A} \eta_{2}\right) n_{2} .
\end{aligned}
$$


In these equations, we have introduced a dimensionless complex dissipation parameter

$$
\mathcal{A}=\frac{k T}{k T+\frac{i \hbar}{2} \Gamma_{\downarrow}}=|\mathcal{A}| e^{i \theta_{\mathcal{A}}} .
$$

We have furthermore used $\delta=\theta_{1}-\theta_{2}$ and $J=|J| e^{i \theta_{J}}$. Omitting the noise terms, we find the equation of motion for the total particle number $n=n_{1}+n_{2}$ :

$$
\begin{aligned}
\dot{n}= & \left(\tilde{\Gamma}_{\uparrow, 1} n_{1}+\tilde{\Gamma}_{\uparrow, 2} n_{2}\right)+2 \frac{\operatorname{Im}(\mathcal{A})}{\operatorname{Re}(\mathcal{A})}\left(\omega_{c, 1} n_{1}+\omega_{c, 2} n_{2}-\bar{\omega}_{c} n\right) \\
& +\frac{4|\mathcal{A}||J|}{\operatorname{Re}(\mathcal{A})} \sin \left(\theta_{J}+\theta_{\mathcal{A}}\right) \sqrt{n_{1} n_{2}} \cos (\delta) .
\end{aligned}
$$

We can draw the following conclusion from this equation:

(1) Equation (B13) implies that a phase difference of

$$
\delta= \begin{cases}0 & \sin \left(\theta_{J}+\theta_{\mathcal{A}}\right)>0 \\ \pi & \text { otherwise, }\end{cases}
$$

maximizes the gain in total particle number. This holds independent of the details of the coupling, condensate frequencies and pumping rates. As a specific example, we consider the case of a real-valued coupling $J$, which implies $\theta_{J}=0$ or $\theta_{J}=\pi$. In typical experimental scenarios, $\theta_{\mathcal{A}}$ is a (small) negative number. For $\theta_{J}=\pi$, we have $\sin \left(\theta_{J}+\theta_{\mathcal{A}}\right)>0$, which means that the symmetric state $(\delta=0)$ maximizes the gain. Based on Eq. (B8), this state also minimizes the energy. For $\theta_{J}=0$, we have the opposite case: the antisymmetric state $(\delta=\pi)$ maximizes the gain and minimizes the energy. This simply means that for real-valued couplings, the minimization of energy and the maximization of gain imply each other. For general coupling constants $J$, however, this is not the case.

(2) For condensates with equal frequencies $\left(\omega_{c, 1}=\omega_{c, 2}\right)$ and a given total particle number $n$, the particle number distribution that maximizes the gain is

$$
\begin{aligned}
n_{1}= & \frac{n}{2}\left[1+\frac{\tilde{\Gamma}_{\uparrow, 1}-\tilde{\Gamma}_{\uparrow, 2}}{\sqrt{\left[\frac{4|\mathcal{A}||J|}{\operatorname{Re}(\mathcal{A})} \sin \left(\theta_{J}+\theta_{\mathcal{A}}\right)\right]^{2}+\left(\tilde{\Gamma}_{\uparrow, 1}-\tilde{\Gamma}_{\uparrow, 2}\right)^{2}}}\right] \\
& \text { with } n_{2}=n-n_{1} .
\end{aligned}
$$

For large coupling constants $|J|$ or equal gain $\left(\tilde{\Gamma}_{\uparrow, 1}=\tilde{\Gamma}_{\uparrow, 2}\right)$, the solution reduces to equal population. In other words, the state $n_{1}=n_{2}=n / 2$ with $\delta=0, \pi$ maximizes the gain. This solution is furthermore a dynamical fixed point of the Josephson equations given in Eqs. (B8)-(B11).

\section{Potential step model}

In this section, we analyze a simplifying model, in which two photon BECs exchange particles via an attractive potential step; see Fig. 7. Using this model, we will gain insight into the physical mechanisms that drive the state selection process between symmetric and antisymmetric wave functions in the photonic Josephson junction.

The starting point is a one-dimensional dissipative Schrödinger equation. We use this equation to find symmetric and antisymmetric wave functions that can be associated to the 0 - and $\pi$-states of a coupled condensate system as discussed in Appendix B.1. The Schrödinger equation now

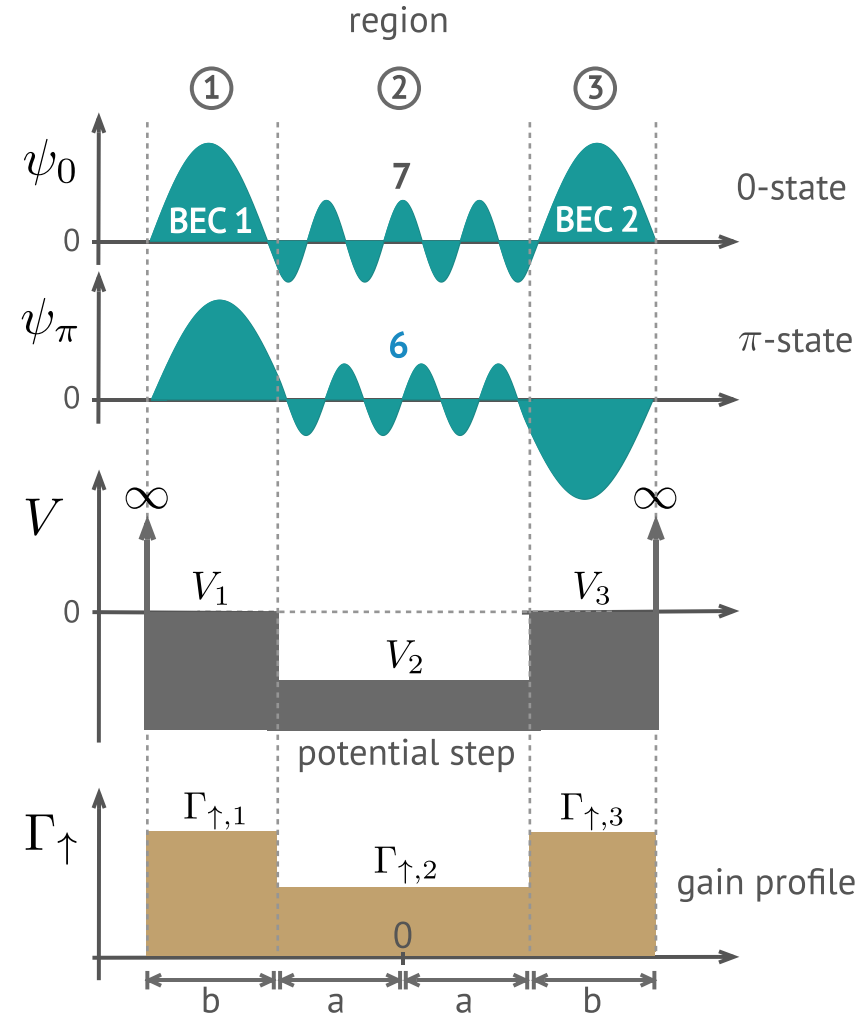

FIG. 7. Potential step model. The model assumes that two BECs exchange particles via an attractive potential step. Depending on the potential energy $V$ and gain $\Gamma_{\uparrow}$ the system selects either 0 - or $\pi$-state. We assume spatially symmetric and piecewise constant functions $V(x), \Gamma_{\uparrow}(x)$.

includes terms related to the kinetic and potential energy:

$$
\begin{aligned}
i \hbar \frac{\partial \psi}{\partial t}= & \hbar \omega_{c} \psi-\frac{\hbar^{2}}{2 m} \frac{\partial^{2} \psi}{\partial x^{2}}+V \psi \\
& +\frac{i \hbar}{2}\left\{\Gamma_{\uparrow}-\Gamma_{\downarrow} \exp \left[\frac{\hbar\left(\dot{\theta}-\omega_{\mathrm{zpl}}\right)}{k T}\right]\right\} \psi .
\end{aligned}
$$

We follow a procedure similar as in Appendix B.1. First, we rewrite the absorption term as

$$
\Gamma_{\downarrow} \exp \left[\frac{\hbar\left(\dot{\theta}-\omega_{\mathrm{zpl}}\right)}{k T}\right]=\tilde{\Gamma}_{\downarrow} \exp \left[\frac{\hbar\left(\dot{\theta}-\omega_{c}\right)}{k T}\right]
$$

with $\tilde{\Gamma}_{\downarrow}=\Gamma_{\downarrow} \exp \left[\hbar\left(\omega_{c}-\omega_{\text {zpl }}\right) / k T\right]$. We assume that all occurring energies stay close to the bare condensate frequency $\omega_{c}$ in the sense that $\hbar\left(\dot{\theta}-\omega_{c}\right) \ll k T$. Under this assumption, we linearize the exponential function in the gain-loss term

$$
\begin{aligned}
i \hbar \frac{\partial \psi}{\partial t}= & \hbar \omega_{c} \psi-\frac{\hbar^{2}}{2 m} \frac{\partial^{2} \psi}{\partial x^{2}}+V \psi \\
& +\frac{i \hbar}{2}\left[\tilde{\Gamma}_{\uparrow}-\tilde{\Gamma}_{\downarrow} \frac{\hbar\left(\dot{\theta}-\omega_{c}\right)}{k T}\right] \psi .
\end{aligned}
$$

In the latter, we have introduced the renormalized gain coefficient $\tilde{\Gamma}_{\uparrow}=\Gamma_{\uparrow}-\tilde{\Gamma}_{\downarrow}$. Assuming slowly varying amplitudes in the wave function $\psi=\sqrt{n} \exp (-i \theta)$, we approximate the phase velocity as $\dot{\theta} \simeq i \dot{\psi} / \psi$. This procedure leads to

$$
i \hbar \frac{\partial \psi}{\partial t}=\hbar \omega_{c} \psi-\frac{\hbar^{2} \mathcal{A}}{2 m} \frac{\partial^{2} \psi}{\partial x^{2}}+\mathcal{A} V \psi+\frac{i \hbar}{2} \mathcal{A} \tilde{\Gamma}_{\uparrow} \psi,
$$


in which we have used the complex dissipation parameter $\mathcal{A}$ as defined in Eq. (B12). Using $\psi(x, t)=\phi(x) \chi(t)$ yields the time-independent Schrödinger equation,

$$
-\frac{\hbar^{2} \mathcal{A}}{2 m} \frac{\partial^{2} \phi}{\partial x^{2}}+\mathcal{A} V \phi+\frac{i \hbar}{2} \mathcal{A} \tilde{\Gamma}_{\uparrow} \phi=\left(E-\hbar \omega_{c}\right) \phi .
$$

The potential and gain profiles are considered symmetric in space, i.e., $V(x)=V(-x)$ and $\tilde{\Gamma}_{\uparrow}(x)=\tilde{\Gamma}_{\uparrow}(-x)$. Furthermore, they are set constant in each of the three regions shown in Fig. 7. For the symmetric state, we use the following ansatz

$$
\phi_{0}(x)= \begin{cases}\sin \left(k_{1}[x+a+b]\right) & -a-b \leqslant x \leqslant-a \\ A \cos \left(k_{2} x\right) & -a \leqslant x \leqslant a \\ -\sin \left(k_{1}[x-a-b]\right) & a \leqslant x \leqslant a+b .\end{cases}
$$

For the antisymmetric state, we choose

$$
\phi_{\pi}(x)= \begin{cases}\sin \left(k_{1}[x+a+b]\right) & -a-b \leqslant x \leqslant-a \\ A \sin \left(k_{2} x\right) & -a \leqslant x \leqslant a \\ \sin \left(k_{1}[x-a-b]\right) & a \leqslant x \leqslant a+b .\end{cases}
$$

Here, the complex wave numbers $k_{i}$ must follow

$$
k_{i}=\hbar^{-1} \sqrt{2 m\left(\frac{E-\hbar \omega_{c}}{\mathcal{A}}-V_{i}-\frac{i \hbar}{2} \tilde{\Gamma}_{\uparrow, i}\right)} .
$$

Continuity conditions for $\phi$ and $\partial \phi / \partial x$ at $x= \pm a$ determine the coefficient $A$. We find

$$
A= \begin{cases}\frac{\sin \left(k_{1} b\right)}{\cos \left(k_{2} a\right)} & (0 \text {-state }) \\ -\frac{\sin \left(k_{1} b\right)}{\sin \left(k_{2} a\right)} & (\pi \text {-state }) .\end{cases}
$$

Furthermore, the continuity conditions deliver an additional relation between the wave numbers. For the two states, these conditions are given by

$$
k_{1} \cot \left(k_{1} b\right)= \begin{cases}k_{2} \tan \left(k_{2} a\right) & (0 \text {-state }) \\ -k_{2} \cot \left(k_{2} a\right) & (\pi \text {-state }) .\end{cases}
$$

Together with Eq. (B23), these equation determine the allowed (complex) energies in the system. Since analytical solutions are not possible, the energy spectrum has to be found numerically. In particular, we are interested in the energies that belong to the kinetic ground state of the condensates, which will be called $E_{0}$ and $E_{\pi}$. These two energies are related to the coupling constant $J$, as defined in Appendix B.1, in the following way:

$$
\begin{aligned}
E_{0}-E_{\pi} & =i \hbar\left(\frac{\dot{\psi}_{0}}{\psi_{0}}-\frac{\dot{\psi}_{\pi}}{\psi_{\pi}}\right) \\
& =\hbar\left(\dot{\theta}_{0}-\dot{\theta}_{\pi}\right)+\frac{i \hbar}{2}\left(\frac{\dot{n}_{0}}{n_{0}}-\frac{\dot{n}_{\pi}}{n_{\pi}}\right) \\
& =2 \hbar \operatorname{Re}(\mathcal{A} J)+i 2 \hbar \operatorname{Im}(\mathcal{A J}) / \operatorname{Re}(\mathcal{A}) .
\end{aligned}
$$

Here, we have used Eqs. (B8) and (B13) with $\tilde{\Gamma}_{\uparrow, 1}=\tilde{\Gamma}_{\uparrow, 2}$, $\omega_{c, 1}=\omega_{c, 2}$ and consequently $n_{1}=n_{2}$. The solution of this equation in terms of the coupling constant is

$$
J=\frac{\operatorname{Re}\left(E_{0}-E_{\pi}\right)+i \operatorname{Re}(\mathcal{A}) \operatorname{Im}\left(E_{0}-E_{\pi}\right)}{2 \hbar \mathcal{A}} .
$$

Figure 8 shows the complex energy difference $\Delta E=E_{0}-E_{\pi}$ as a function of the potential step depth $V_{2}$ for three parameter sets, which represent limiting cases for the system. For positive imaginary components, i.e., $\operatorname{Im}(\Delta E)>0$, the 0 -state system has a larger gain than the $\pi$-state. Consequently, the system will predominantly realize the 0 -state. For negative $\operatorname{Im}(\Delta E)$, the system chooses the $\pi$-state. For all parameter sets, the gain periodically switches sign as the depth of the potential step is increased.

For large gain gradients $\Gamma_{\uparrow, 1}-\Gamma_{\uparrow, 2}$ (left column) or high temperatures $T$ (right column), the system primarily maximizes the spatial overlap with the high gain region. The latter is reflected by the fact that the gain curve $\operatorname{Im}(\Delta E)$ correlates with the quantity $\bar{\Gamma}_{\uparrow}^{0}-\bar{\Gamma}_{\uparrow}^{\pi}$, as shown in the third row of Fig. 8. Here, $\bar{\Gamma}_{\uparrow}^{0, \pi}=\int_{-a-b}^{-+a+b} \Gamma_{\uparrow}\left|\psi_{0, \pi}\right|^{2} d x$ (with normalized wave functions $\psi_{0, \pi}$ ) is the spatially averaged gain. This quantity is positive, if the 0-state has a larger overlap with the high gain regions than the $\pi$-state (and is negative otherwise). For small gradients $\Gamma_{\uparrow, 1}-\Gamma_{\uparrow, 2}$ or low temperatures $T$ (middle column), the system primarily minimizes the energy, which is reflected by the fact that the gain curve $\operatorname{Im}(\Delta E)$ now correlates with the energy curve $\operatorname{Re}(\Delta E)$. This behavior can be understood as a consequence of the thermalization process induced by the KS gain-loss scheme.

\section{3. $N$ coupled condensates}

Equations (B8), (B9), and (B13) can readily be generalized to $N$ coupled condensates with coupling constants $J_{i j}$. In the following, we set all condensate frequencies and pumping rates equal: $\omega_{c, i}=\omega_{c}$ and $\Gamma_{\uparrow, i}=\Gamma_{\uparrow}$. For the phase velocity, we find:

$$
\begin{aligned}
\dot{\theta}_{i}= & \omega_{c}+\frac{1}{2} \operatorname{Im}(\mathcal{A})\left(\frac{\dot{n}_{i}}{n_{i}}-\tilde{\Gamma}_{\uparrow}\right) \\
& +\sum_{j}|\mathcal{A}|\left|J_{i j}\right| \sqrt{n_{j} / n_{i}} \cos \left(\theta_{J_{i j}}+\theta_{\mathcal{A}}+\theta_{i}-\theta_{j}\right) .
\end{aligned}
$$

For the particle number evolution, we obtain

$$
\dot{n}_{i}=\tilde{\Gamma}_{\uparrow} n_{i}+\sum_{j} \frac{2|\mathcal{A}|\left|J_{i j}\right|}{\operatorname{Re}(\mathcal{A})} \sqrt{n_{i} n_{j}} \sin \left(\theta_{J_{i j}}+\theta_{\mathcal{A}}+\theta_{i}-\theta_{j}\right) .
$$

The total particle number gain $\dot{n}=\sum_{i} \dot{n}_{i}$ is given by

$$
\begin{aligned}
& \dot{n}=\sum_{i} \tilde{\Gamma}_{\uparrow} n_{i}+\sum_{i, j} \frac{2|\mathcal{A}|\left|J_{i j}\right|}{\operatorname{Re}(\mathcal{A})} \sqrt{n_{i} n_{j}} \sin \left(\theta_{J_{i j}}+\theta_{\mathcal{A}}+\theta_{i}-\theta_{j}\right) \\
& =\sum_{i} \tilde{\Gamma}_{\uparrow} n_{i}+\sum_{i>j} \frac{4|\mathcal{A}|\left|J_{i j}\right|}{\operatorname{Re}(\mathcal{A})} \sqrt{n_{i} n_{j}} \sin \left(\theta_{J_{i j}}+\theta_{\mathcal{A}}\right) \cos \left(\theta_{i}-\theta_{j}\right),
\end{aligned}
$$

which can be written in the form

$$
\dot{n}=\sum_{i} \tilde{\Gamma}_{\uparrow} n_{i}-\sum_{i>j} J_{i j}^{X Y} \cos \left(\theta_{i}-\theta_{j}\right) .
$$

Here, $J_{i j}^{X Y}=-4|\mathcal{A}|\left|J_{i j}\right|(\operatorname{Re} \mathcal{A})^{-1} \sqrt{n_{i} n_{j}} \sin \left(\theta_{J_{i j}}+\theta_{\mathcal{A}}\right)$ describes an effective coupling constant. Assuming that all condensates have the same number of particles, only the phases of the 


$$
\begin{aligned}
T & =300 \mathrm{~K} \\
\Gamma_{\uparrow, 1} & =75 \mathrm{GHz} \\
\Gamma_{\uparrow, 2} & =50 \mathrm{GHz} \\
\tilde{\Gamma}_{\downarrow} & =50 \mathrm{GHz}
\end{aligned}
$$

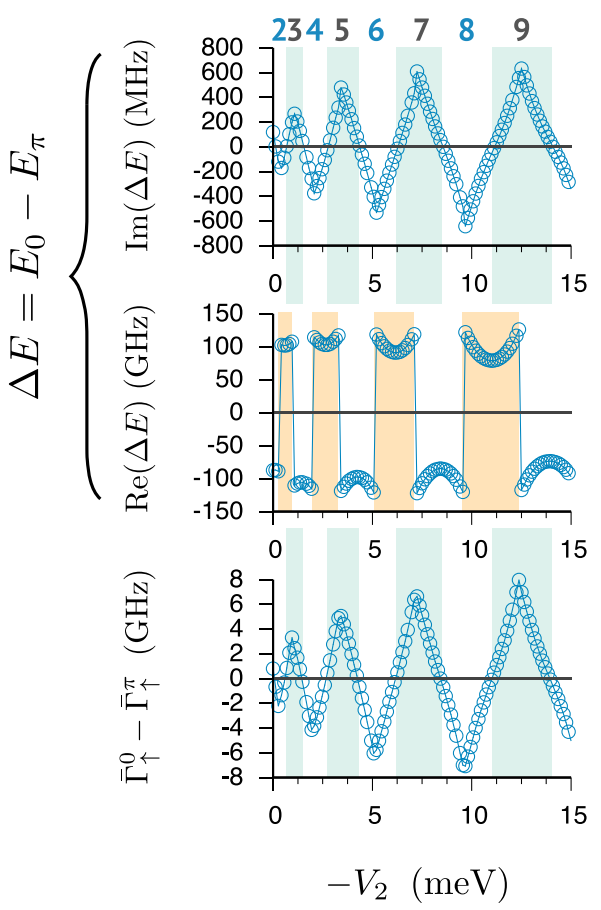

state maximizes spatial overlap with high gain regions

$$
\begin{aligned}
T & =300 \mathrm{~K} \\
\Gamma_{\uparrow, 1} & =51 \mathrm{GHz} \\
\Gamma_{\uparrow, 2} & =50 \mathrm{GHz} \\
\tilde{\Gamma}_{\downarrow} & =50 \mathrm{GHz}
\end{aligned}
$$

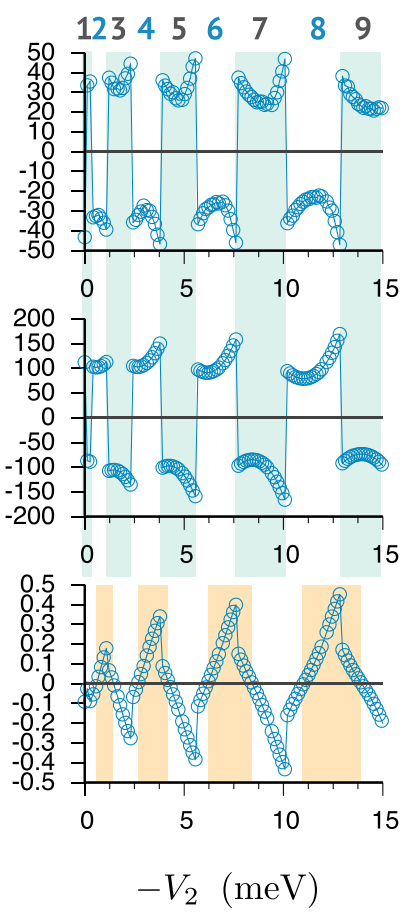

state minimizes energy

$$
\begin{aligned}
T & =10000 \mathrm{~K} \\
\Gamma_{\uparrow, 1} & =51 \mathrm{GHz} \\
\Gamma_{\uparrow, 2} & =50 \mathrm{GHz} \\
\tilde{\Gamma}_{\downarrow} & =50 \mathrm{GHz}
\end{aligned}
$$

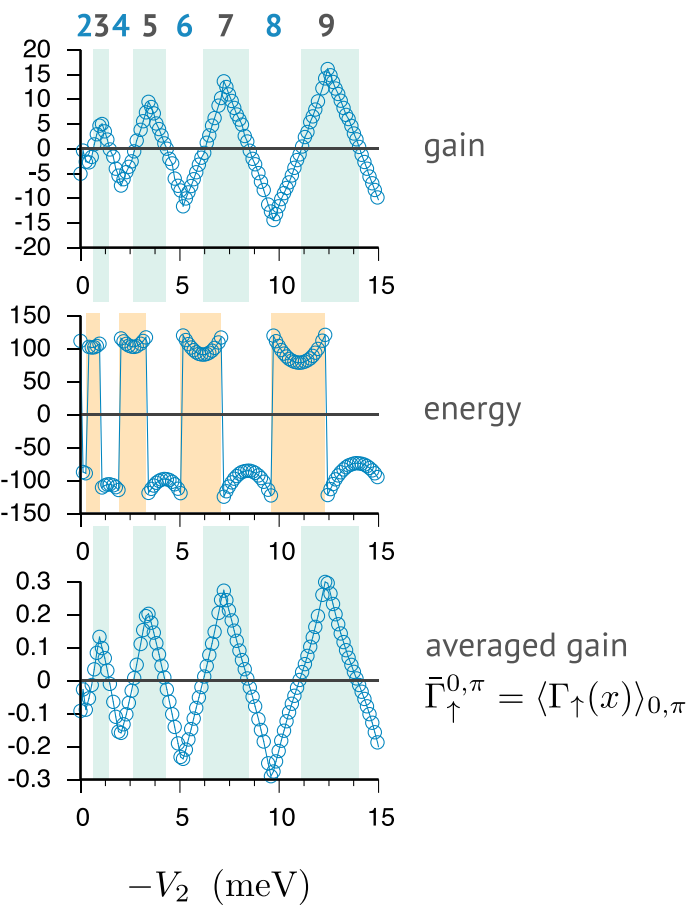

state maximizes spatial overlap with high gain regions

FIG. 8. Complex energy difference $\Delta E=E_{0}-E_{\pi}$ as a function of potential step depth $V_{2}$ for three parameter regimes. For positive $\operatorname{Im}(\Delta E)$, the system predominantly realizes the 0 -state. For negative $\operatorname{Im}(\Delta E)$, the system chooses to be in the $\pi$-state. For all parameter sets, the gain periodically switches sign as the depth of the potential step is increased. For large gain gradients $\Gamma_{\uparrow, 1}-\Gamma_{\uparrow, 2}$ (left column) or high temperatures $T$ (right column), the system maximizes the spatial overlap with the high gain regions, as indicated by the quantity $\bar{\Gamma}_{\uparrow}^{0}-\bar{\Gamma}_{\uparrow}^{\pi}$ (see text). For small gradients or low temperatures (middle column), the system minimizes (the real part of) the energy. Further parameters: $m=6.5 \times 10^{-36} \mathrm{~kg}, a=9 \mu \mathrm{m}, b=7 \mu \mathrm{m}$.

condensates remain free. In this case, the second term in Eq. (B34) exactly corresponds to the Hamiltonian of a classical XY model. The phase configuration $\left\{\theta_{i}\right\}$ that maximizes the gain, minimizes the energy of the simulated XY Hamiltonian. This implies that the coupled BECs system can be used to sample low energy configurations of the simulated XY Hamiltonian. In general, the assumed equality of particle numbers is not automatically guaranteed and may require a feedback scheme [16] (and references therein). Furthermore, it is not guaranteed that the system relaxes to a single state as the configuration that maximizes the gain is not necessarily a fix point of the Josephson equations.

\section{Spin-glass simulation with photonic Josephson junctions-Numerical results}

In Fig. 5 we use numerical simulations to investigate the dynamics of the condensation process on a 3-regular planar graph. These simulations are based on numerical solutions of a two-dimensional, stochastic and dissipative Schrödinger equation:

$$
\begin{aligned}
i \hbar \frac{\partial \psi}{\partial t}= & \hbar \omega_{c} \psi-\frac{\hbar^{2}}{2 m}\left(\frac{\partial^{2} \psi}{\partial x^{2}}+\frac{\partial^{2} \psi}{\partial y^{2}}\right)+V \psi \\
& +\frac{i \hbar}{2}\left\{\Gamma_{\uparrow}-\Gamma_{\downarrow} \exp \left[\frac{\hbar\left(\dot{\theta}-\omega_{\mathrm{zpl}}\right)}{k T}\right]-\Gamma_{2}|\psi|^{2}\right\} \psi+\hbar \eta
\end{aligned}
$$

This equation is an extension of Eq. (B16) to the twodimensional domain. It includes an additional term related which allows us to model gain saturation in the system. Moreover, we added the noise function $\eta=\eta(t)$. We perform the same steps and approximations as in Appendix B.2 to obtain

$$
\begin{aligned}
i \hbar \frac{\partial \psi}{\partial t}= & \hbar \omega_{c} \psi-\frac{\hbar^{2} \mathcal{A}}{2 m}\left(\frac{\partial^{2} \psi}{\partial x^{2}}+\frac{\partial^{2} \psi}{\partial y^{2}}\right)+\mathcal{A} V \psi \\
& +\frac{i \hbar}{2} \mathcal{A}\left(\tilde{\Gamma}_{\uparrow}-\Gamma_{2}|\psi|^{2}\right) \psi+\hbar \mathcal{A} \eta
\end{aligned}
$$


Numerical solutions of this equation are obtained with the Runge-Kutta method (4th order) with constant time steps. The open source software library ViennaCL is used to perform the computations on a fast GPU. We have verified that our numerical results reproduce known analytical results in a series of test cases. The ground-state problem shown in Fig. 5 is mapped onto the potential landscape in the microcavity system by introducing a triangular lattice potential, in which the lattice sites are connected by attractive step potentials. To simulate antiferromagnetic couplings $\left(J_{i j}^{X Y}=-1\right)$, the depth of the steps is chosen to favour the formation of $\pi$-states. The gain profile $\Gamma_{\uparrow}(x, y)$ follows the lattice geometry. The spatial profile of the loss (absorption) is assumed to be homogeneous $\Gamma_{\downarrow}(x, y)=1 \mathrm{THz}$. We furthermore assume $T=300 \mathrm{~K}$, which sets the dissipation parameter $\mathcal{A}$ given in Eq. (B12). Condensate frequency and zero-phonon line are considered equal: $\omega_{c}=\omega_{\mathrm{zpl}}$. The effective photon mass in the simulation is $m=6.5 \times 10^{-36} \mathrm{~kg}$.
[1] A. Lucas, Ising formulations of many NP problems, Front. Phys. 12, 1 (2014).

[2] F. Barahona, On the computational complexity of Ising spin glass models, J. Phys. A-Math. Gen. 15, 3241 (1982).

[3] T. Cubitt and A. Montanaro, Complexity classification of local Hamiltonian problems, SIAM J. Comput. 45, 268 (2016).

[4] M. W. Johnson, M. H. S. Amin, S. Gildert, T. Lanting, F. Hamze, N. Dickson, R. Harris, A. J. Berkley, J. Johansson, P. Bunyik et al., Quantum annealing with manufactured spins, Nature (London) 473, 194 (2011).

[5] S. Boixo, T. F. Rønnow, S. V. Isakov, Z. Wang, D. Wecker, D. A. Lidar, J. M. Martinis, and M. Troyer, Evidence for quantum annealing with more than one hundred qubits, Nat. Phys. 10, 218 (2014).

[6] A. Marandi, Z. Wang, K. Takata, R. L. Byer, and Y. Yamamoto, Network of time-multiplexed optical parametric oscillators as a coherent Ising machine, Nat. Photonics 8, 937 (2014).

[7] P. L. McMahon, A. Marandi, Y. Haribara, R. Hamerly, C. Langrock, S. Tamate, T. Inagaki, H. Takesue, S. Utsonomiya, K. Aihara et al., A fully programmable 100-spin coherent Ising machine with all-to-all connections, Science 354, 614 (2016).

[8] T. Inagaki, K. Inaba, R. Hamerly, K. Inoue, Y. Yamamoto, and H. Takesue, Large-scale Ising spin network based on degenerate optical parametric oscillators, Nat. Photonics 10, 415 (2016).

[9] A. D. King, J. Carrasquilla, J. Raymond, I. Ozfidan, E. Andriyash, A. Berkley, M. Reis, T. Lanting, R. Harris, F. Altomare et al., Observation of topological phenomena in a programmable lattice of 1, 800 qubits, Nature (London) 560, 456 (2018).

[10] M. Nixon, E. Ronen, A. A. Friesem, and N. Davidson, Observing Geometric Frustration With Thousands of Coupled Lasers, Phys. Rev. Lett. 110, 184102 (2013).

[11] J. Struck, M. Weinberg, C. Ölschläger, P. Windpassinger, J. Simonet, K. Sengstock, R. Höppner, P. Hauke, A. Eckardt, M. Lewenstein, and L. Mathey, Engineering Ising-XY spin-models in a triangular lattice using tunable artificial gauge fields, Nat. Phys. 9, 738 (2013).

[12] H. Ohadi, R. L. Gregory, T. Freegarde, Y. G. Rubo, A. V. Kavokin, N. G. Berloff, and P. G. Lagoudakis, Nontrivial Phase Coupling in Polariton Multiplets, Phys. Rev. X 6, 031032 (2016).

[13] N. G. Berloff, M. Silva, K. Kalinin, A. Askitopoulos, J. D. Töpfer, P. Cilibrizzi, W. Langbein, and P. G. Lagoudakis, Realizing the classical XY Hamiltonian in polariton simulators, Nat. Mater. 16, 1120 (2017).

[14] K. P. Kalinin and N. G. Berloff, Simulating Ising and n-State Planar Potts Models and External Fields With Nonequilibrium Condensates, Phys. Rev. Lett. 121, 235302 (2018).
[15] H. Ohadi, Y. del Valle-Inclan Redondo, A. J. Ramsay, Z. Hatzopoulos, T. C. H. Liew, P. R. Eastham, P. G. Savvidis, and J. J. Baumberg, Synchronization crossover of polariton condensates in weakly disordered lattices, Phys. Rev. B 97, 195109 (2018).

[16] K. P. Kalinin and N. G. Berloff, Toward arbitrary control of lattice interactions in nonequilibrium condensates, Adv. Quantum Technol. 3, 1900065 (2019).

[17] S. Alyatkin, J. D. Töpfer, A. Askitopoulos, H. Sigurdsson, and P. G. Lagoudakis, Optical Control of Couplings in Polariton Condensate Lattices, Phys. Rev. Lett. 124, 207402 (2020).

[18] B. D. Josephson, Possible new effects in superconductive tunnelling, Phys. Lett. 1, 251 (1962).

[19] E. C. Gingrich, B. M. Niedzielski, J. A. Glick, Y. Wang, D. L. Miller, R. Loloee, W. P. Pratt Jr., and N. O. Birge et al., Controllable $0-\pi$ Josephson junctions containing a ferromagnetic spin valve, Nat. Phys. 12, 564 (2016).

[20] F. S. Cataliotti, S. Burger, C. Fort, P. Maddaloni, F. Minardi, A. Trombettoni, A. Smerzi, and M. Inguscio, Josephson junction arrays with Bose-Einstein condensates, Science 293, 843 (2001).

[21] C. W. Lai, N. Y. Kim, S. Utsunomiya, G. Roumpos, H. Deng, M. D. Fraser, T. Byrnes, P. Recher, N. Kumada, T. Fujisawa, and Y. Yamamoto, Coherent zero-state and $\pi$-state in an excitonpolariton condensate array, Nature (London) 450, 529 (2007).

[22] K. G. Lagoudakis, B. Pietka, M. Wouters, R. André, and B. Deveaud-Plédran, Coherent Oscillations in an ExcitonPolariton Josephson Junction, Phys. Rev. Lett. 105, 120403 (2010).

[23] M. Abbarchi, A. Amo, V. G. Sala, D. D. Solnyshkov, H. Flayac, L. Ferrier, I. Sagnes, E. Galopin, A. Lemaître, G. Malpuech, and J. Bloch, Macroscopic quantum self-trapping and Josephson oscillations of exciton polaritons, Nat. Phys. 9, 275 (2013).

[24] A. F. Adiyatullin, M. D. Anderson, H. Flayac, M. T. PortellaOberli, F. Jabeen, C. Ouellet-Plamondon, G. C. Sallen, and B. Deveaud, Periodic squeezing in a polariton Josephson junction, Nat. Commun. 8, 1329 (2017).

[25] J. Klaers, F. Vewinger, and M. Weitz, Thermalization of a twodimensional photonic gas in a 'white wall' photon box, Nat. Phys. 6, 512 (2010).

[26] J. Klaers, J. Schmitt, F. Vewinger, and M. Weitz, Bose-Einstein condensation of photons in an optical microcavity, Nature (London) 468, 545 (2010).

[27] P. Kirton, J. Keeling, Nonequilibrium Model of Photon Condensation, Phys. Rev. Lett. 111, 100404 (2013).

[28] D. Dung, C. Kurtscheid, T. Damm, J. Schmitt, F. Vewinger, M. Weitz, and J. Klaers, Variable potentials for thermalized light and coupled condensates, Nat. Photonics 11, 565 (2017). 
[29] B. T. Walker, L. C. Flatten, H. J. Hesten, F. Mintert, D. Hunger, A. A. P. Trichet, J. M. Smith, and R. A. Nyman, Drivendissipative non-equilibrium Bose-Einstein condensation of less than ten photons, Nat. Phys. 14, 1173 (2018).

[30] S. Greveling, K. L. Perrier, and D. van Oosten, Density distribution of a Bose-Einstein condensate of photons in a dye-filled microcavity, Phys. Rev. A 98, 013810 (2018).

[31] V. N. Gladilin and M. Wouters, Classical field model for arrays of photon condensates, Phys. Rev. A 101, 043814 (2020).

[32] C. Kurtscheid, D. Dung, E. Busley, F. Vewinger, A. Rosch, and M. Weitz, Thermally condensing photons into a coherently split state of light, Science 366, 894 (2019).

[33] R. Balili, V. Hartwell, D. Snoke, L. Pfeiffer, and K. West, Bose-Einstein condensation of microcavity polaritons in a trap, Science 316, 1007 (2007).

[34] M. M. de Lima, M. van der Poel, P. V. Santos, and J. M. Hvam, Phonon-Induced Polariton Superlattices, Phys. Rev. Lett. 97, 045501 (2006).

[35] M. D. Fraser, H. Tan, and C. Jagadish, Selective confinement of macroscopic long-lifetime exciton and trion populations, Phys. Rev. B 84, 245318 (2011).
[36] E. Wertz, L. Ferrier, D. D. Solnyshkov, R. Johne, D. Sanvitto, A. Lemaître, I. Sagnes, R. Grousson, A. V. Kavokin, P. Senellart, G. Malpuech, and J. Bloch. Spontaneous formation and optical manipulation of extended polariton condensates, Nat. Phys. 6, 860 (2010).

[37] E. Moreau, I. Robert, J. M. Gérard, I. Abram, L. Manin, and V. Thierry-Mieg, Single-mode solid-state single photon source based on isolated quantum dots in pillar microcavities, Appl. Phys. Lett. 79, 2865 (2001).

[38] C. Kurtscheid, D. Dung, A. Redmann, E. Busley, J. Klaers, F. Vewinger, J. Schmitt, and M. Weitz, Realizing arbitrary trapping potentials for light via direct laser writing of mirror surface profiles, Europhys. Lett. 130, 54001 (2020).

[39] J. D. Töpfer, I. Chatzopoulos, H. Sigurdsson, T. Cookson, Y. G. Rubo, and P. G. Lagoudakis, Engineering spatial coherence in lattices of polariton condensates, Optica 8, 106 (2021).

[40] J. Klaers, J. Schmitt, T. Damm, F. Vewinger, and M. Weitz, Statistical Physics of Bose-Einstein-Condensed Light in a Dye Microcavity, Phys. Rev. Lett. 108, 160403 (2012). 